\title{
Low-Complexity Super-Resolution Detection for Range-Vital Doppler Estimation FMCW Radar
}

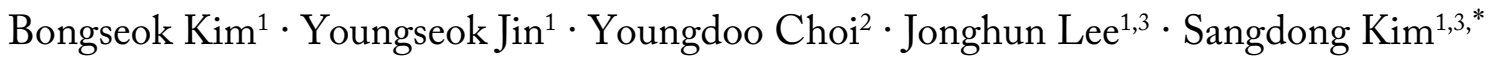

\begin{abstract}
This paper proposes low-complexity super-resolution detection for range-vital Doppler estimation frequency-modulated continuous wave (FMCW) radar. In regards to vital radar, and in order to estimate joint range and vital Doppler information such as the human heartbeat and respiration, two-dimensional (2D) detection algorithms such as 2D-FFT (fast Fourier transform) and 2D-MUSIC (multiple signal classification) are required. However, due to the high complexity of $2 \mathrm{D}$ full-search algorithms, it is difficult to apply this process to lowcost vital FMCW systems. In this paper, we propose a method to estimate the range and vital Doppler parameters by using 1D-FFT and 1D-MUSIC algorithms, respectively. Among 1D-FFT outputs for range detection, we extract 1D-FFT results based solely on human target information with phase variation of respiration for each chirp; subsequently, the 1D-MUSIC algorithm is employed to obtain accurate vital Doppler results. By reducing the dimensions of the estimation algorithm from $2 \mathrm{D}$ to $1 \mathrm{D}$, the computational burden is reduced. In order to verify the performance of the proposed algorithm, we compare the Monte Carlo simulation and root-mean-square error results. The simulation and experiment results show that the complexity of the proposed algorithm is significantly lower than that of an algorithm detecting signals in several regions.
\end{abstract}

Key Words: FFT, Low Complexity, MUSIC, Super-Resolution, Vital FMCW Radar.

\section{INTRODUCTION}

Recently, microwave Doppler radar has been utilized to detect movements of the human body, such as respiration and heartbeat. The detection of movements is used for various applications such as life detection in earthquake events [1] and localization monitoring of enemies in military applications [2]. The microwave radar sensing system radiates a single tone continuous-wave (CW) signal [3-8]; the reflected signal is then demodulated in the receiver. The $\mathrm{CW}$ radar can obtain movement information from the phase change of the time-varying chest- wall position. The received signal with the demodulated phase is proportional to the chest-wall position information due to respiration and heartbeat.

While CW radar extracts only vital Doppler parameters, frequency-modulated continuous-wave (FMCW) radar can extract multiple parameters, such as distance or Doppler. In the case of FMCW radar, fast Fourier transform (FFT)-based algorithms are used in conventional FMCW radar systems to acquire multiple parameters. However, the FFT-based parameter estimator has considerably low resolution and accuracy [9]. Since the resolution of FFT is low, super-resolution algorithms

Manuscript received September 10, 2020 ; Revised February 1, 2021 ; Accepted March 2, 2021. (ID No. 20200910-139J)

${ }^{1}$ Division of Automotive Technology, Daegu Gyeongbuk Institute of Science \& Technology (DGIST), Daegu, Korea.

${ }^{2}$ ROK Navy Academy, Jinhae, Korea.

${ }^{3}$ Interdisciplinary Engineering, Daegu Gyeongbuk Institute of Science Technology (DGIST), Daegu, Korea.

"Corresponding Author: Sangdong Kim (e-mail: kimsd728@dgist.ac.kr)

This is an Open-Access article distributed under the terms of the Creative Commons Attribution Non-Commercial License (http://creativecommons.org/licenses/by-nc/4.0) which permits unrestricted non-commercial use, distribution, and reproduction in any medium, provided the original work is properly cited.

(c) Copyright The Korean Institute of Electromagnetic Engineering and Science. 
such as multiple signal classification (MUSIC) and estimation of signal parameters via rotational invariance techniques (ESPRIT) have been used for vital Doppler estimation. In specific, the MUSIC algorithm utilizes the orthogonality between the signal subspace and the noise subspace. In this way, the algorithm can estimate the super-resolution frequency for the parameters; however, compared to low complexity FFT-based methods, this super-resolution method involves much higher complexity. For this reason, it is difficult to apply the super-resolution method to low-cost and real-time vital FMCW systems, and low-complexity two-dimensional (2D) high-resolution algorithms are subsequently required for compact vital radar.

To date, conventional research has been processed on superresolution algorithms to reduce complexity. A low-complexity super-resolution algorithm without eigenvalue decomposition (EVD) was proposed [10]. The EVD is an essential function to distinguish signal eigenvectors from noise eigenvectors. In this method, the effect of signal eigenvectors is minimized by performing the inverse function as opposed to the EVD. It has similar resolution performance to super-resolution algorithms such as MUSIC. However, a 2D estimation technique is required to simultaneously estimate the respiration signal and position. It still possesses a high complexity to perform multiple parameter estimation for the purpose of obtaining multiple parameters. Therefore, the low-complexity super-resolution algorithm without EVD [10] is difficult to apply to a real-time system. A lowcomplexity ESPRIT algorithm using reduced-dimension (RD) transformation in a monostatic MIMO radar [11] was proposed. This research assumed that the transmit/receive module is on a radar. In [11], RD transformation was generated by rearranging the Kronecker product of the transmitted steering vector and the received steering vector. However, the low-complexity ESPRIT algorithm using $\mathrm{RD}$ transformation in a monostatic MIMO radar [11] has a limitation wherein it can only be operated in the monostatic MIMO radar. An efficient subspacebased algorithm technique for L-shaped array radar [12] was proposed. The azimuth and elevation direction antenna array structure is the L structure. In [12], a new matrix with information for each parameter was generated by using cross correlation between the received signal in the azimuth direction and the received signal in the elevation direction. The cost function of this matrix with the steering vector of the interested signal obtained a 2D direction-of-arrival (DOA) result using maximum value. However, the efficient subspace-based algorithm technique for L-shaped array radar [12] also has a limitation wherein it can only be operated in the 2D L-shaped antenna array structure. Since conventional research is still high complexity and may have disadvantages of operating solely under specific conditions, these research methods have limitations in their application for vital radars. For low complexity, the pro- posed algorithm needs a method to reduce the complexity by extracting only the interested signal. This method reduces complexity by using Doppler processing of only the interested signal in the distance $1 \mathrm{D}$ estimation result.

This paper proposes a low-complexity FFT-MUSIC vital Doppler estimator based on target detection for contactless vital FMCW radar. The proposed method uses FFT to estimate the distance parameter; chirp data of each range bin, with the exception of clutter with stationary phase information, are subsequently used to detect the phase variation and extract the vital signal. After range peak detection, the 1D-MUSIC algorithm is employed to obtain vital Doppler results using only human FFT results. Thus, when compared with full-search super-resolution algorithms, the proposed algorithm reduces the complexity.

The remainder of this paper is organized as follows. Section II presents the signal model of the distance and the vital Doppler for the vital FMCW radar. Section III presents the proposed low complexity MUSIC vital Doppler estimator based on target detection for contactless vital FMCW radar. The complexity of each algorithm is also analyzed in this section. Section IV details the simulations for the various environments. In Section V, experimental results are provided. Finally, conclusions are given in Section VI.

\section{SIGNAL MODEL}

This section addresses the system models of the vital FMCW radar. The vital FMCW radar can estimate parameters such as distance and vital Doppler of humans. Particularly, vital Doppler signals, such as those for respiration and heartbeat, are estimated from the phase information of a reflected signal. An FMCW transmitted (TX) signal is reflected from multiple humans. The reflected signal is changed into a beat signal as a sinusoidal signal at the received (RX) part. The sinusoidal signal's frequency is proportional to the time delay from the human subject. The system model of FMCW radar is considered for multiple humans. The vital FMCW TX signal in Fig. 1 is denoted by $\boldsymbol{s}_{\mathrm{tx}}(\boldsymbol{t})$ and it is represented by

$$
s_{\mathrm{tx}}(t)=\sum_{l=0}^{L-1} s_{0}\left(t-l T_{F}\right)
$$

where $L$ indicates the number of FMCW chirp signals, and $T_{F}$ denotes the total duration of chirp symbol and idle period, i.e., $T_{F}=T+T_{i}, T$ is the duration of the chirp symbol, and $T_{i}$ represents the duration of the idle period.

The vital FMCW chirp symbol $\boldsymbol{s}_{\mathbf{0}}(\boldsymbol{t})$ is composed of

$$
s_{0}(t)=\left\{\begin{array}{cc}
\exp \left(j\left(2 \pi f_{0} t+\frac{\mu t^{2}}{2}\right)\right), & \text { for } 0 \leq t<T \\
0, & \text { elsewhere }
\end{array}\right.
$$




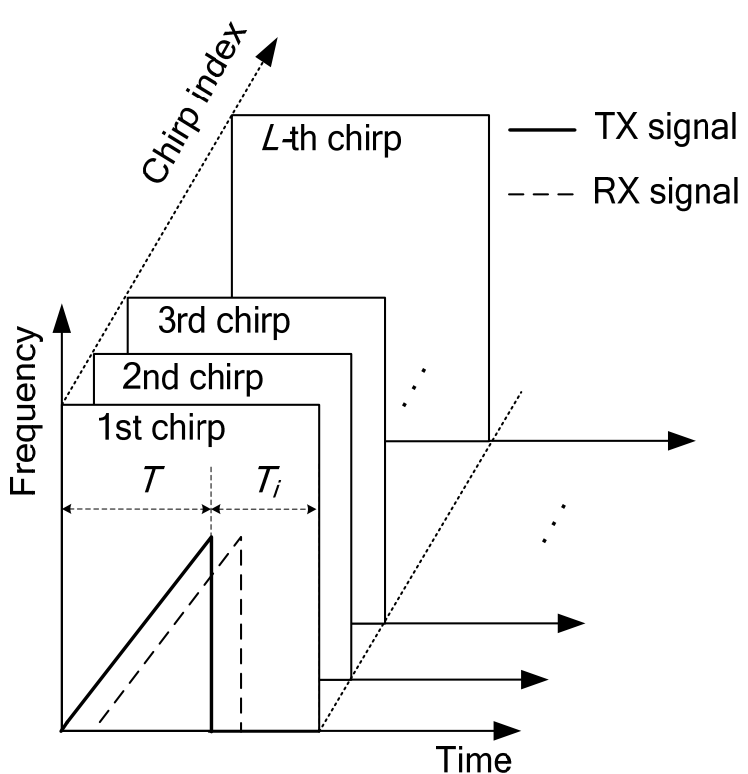

Fig. 1. Waveforms of FMCW radar.

where $f_{0}$ indicates the initial frequency, $\mu$ is the frequency slope of the FMCW chirp symbol according to time, i.e., $\mu=2 \pi f_{B} / T$, and $f_{B W}$ represents the bandwidth of the FMCW signal.

For vital Doppler estimation of $M$ targets, the definition of body movement is needed to determine the targets' fixed distance $\boldsymbol{d}_{\mathbf{0}, \boldsymbol{m}}$ and the time-varying distance $\boldsymbol{x}_{\boldsymbol{m}}(\boldsymbol{t})$ of the $m$-th target, such as heartbeat signal and chest displacement by respiration. The time-varying distance between the radar and the human subject is represented by

$$
d_{m}(t)=d_{0, m}(t)+x_{m}(t)
$$

where $x_{m}(t)=x_{m, \mathrm{r}}(t)+x_{m, \mathrm{~h}}(t) ; x_{m, \mathrm{r}}(t)$ and $x_{m, \mathrm{~h}}(t)$ represent the $m$-th human's body motions by respiration and heartbeat, respectively. The time-varying distance $\boldsymbol{x}_{\boldsymbol{m}}(\boldsymbol{t})$ is composed of movements of

$$
\begin{aligned}
x_{m}(t) & =\underbrace{x_{m, \mathrm{r}}(t)}_{\text {respiration }}+\underbrace{x_{m, \mathrm{~h}}(t)}_{\text {heartbeat }} \\
& =\underbrace{a_{m, \mathrm{r}} \sin \left(2 \pi f_{m, \mathrm{r}} t\right)}_{x_{m, \mathrm{r}}(t)}+\underbrace{a_{m, \mathrm{~h}} \sin \left(2 \pi f_{m, \mathrm{~h}} t\right)}_{x_{m, \mathrm{~h}}(t)}
\end{aligned}
$$

where $\boldsymbol{a}_{\boldsymbol{m}, \mathbf{r}}$ and $\boldsymbol{a}_{\boldsymbol{m}, \mathbf{h}}$ are the amplitude of the respiration and the heartbeat, respectively, and $\boldsymbol{f}_{\boldsymbol{m}, \mathbf{r}}$ and $\boldsymbol{f}_{\boldsymbol{m}, \mathbf{h}}$ denote the frequency of respiration and heartbeat, respectively. The RX signal from the $m$-th human is denoted by $\widetilde{\boldsymbol{y}}_{\boldsymbol{l}}(\boldsymbol{t})$ and obtained with time delay $\boldsymbol{\tau}_{\boldsymbol{m}}$ in (5), at the bottom of this page, where $\widetilde{\boldsymbol{a}}_{\boldsymbol{m}}$ is the $m$-th target's complex amplitude, $\boldsymbol{x}_{\boldsymbol{m}, \mathbf{r}, \boldsymbol{p}}(\boldsymbol{t})$ are the $p$-th respiration harmonic components for the $m$-th human, $\theta$ denotes the residual phase, $\lambda$ is the wavelength and $\omega(t)$ is the additive white Gaussian noise (AWGN) signal. In order to reduce the complexity of the FMCW radar, a de-chirping method is utilized. The de-chirping method defines the multiplication technique of the conjugation FMCW TX signals $\boldsymbol{s}_{\mathrm{tx}}^{*}(\boldsymbol{t})$ and $\widetilde{\boldsymbol{y}}_{l}(\boldsymbol{t})$; the beat signal $\boldsymbol{y}_{\boldsymbol{l}}(\boldsymbol{t})$ at the $l$-th chirp symbol is expressed as

$$
y_{l}(t)=\tilde{y}_{l}(t) s_{\mathrm{tx}}^{*}(t)
$$

After an analog-to-digital converter (ADC) with $f_{s}=1 / T_{s}$ where $f_{s}$ is the sampling frequency and $T_{s}$ is sampling time interval and $N_{s}$ is the number of samples, the converted FMCW signal is denoted by $\boldsymbol{y}_{l}[\boldsymbol{n}]$ and it is expressed in (7), at the top of this page, where $a_{m}=\tilde{a}_{m} \exp \left(\omega_{s} \tau_{m}-\mu \tau_{m}{ }^{2} / 2\right)$ and $p$ is the index of respiration harmonic, i.e., the case of $p=1$ means the main respiration signal and the cases of $p=2,3, \ldots, P$ denotes the respiration harmonic components. From (7), the vector form $\boldsymbol{y}_{\boldsymbol{l}}[\boldsymbol{n}]$ is denoted by $\boldsymbol{y}_{\boldsymbol{l}}$ and expressed as:

$$
\boldsymbol{y}_{l}=\left[y_{l}[0], y_{l}[1], \ldots, y_{l}\left[N_{s}-1\right]\right]^{\mathrm{T}}
$$

The vector form $\boldsymbol{y}_{\boldsymbol{l}}$ is rewritten by the range, the vital Doppler, and the DOA terms, respectively, as follows:

$$
\boldsymbol{y}_{l}=\alpha V_{l} \boldsymbol{r}+\boldsymbol{\omega}
$$

where $\boldsymbol{\alpha}, \boldsymbol{r}$, and $\boldsymbol{\omega}$ are vectors composed of amplitude, range, and noise terms, respectively, i.e., $\quad \boldsymbol{\alpha}=\left[a_{0}, a_{1}, \ldots, a_{N-1}\right]^{\mathrm{T}}$, $\boldsymbol{r}=\left[r\left(\tau_{0}\right), r\left(\tau_{1}\right), \ldots, r\left(\tau_{M}\right)\right] \quad$ and $\quad \boldsymbol{\omega}=\left[\omega_{0}, \omega_{1}, \ldots, \omega_{N-1}\right]^{\mathrm{T}}$ where $\boldsymbol{r}\left(\boldsymbol{\tau}_{\boldsymbol{m}}\right)$ is the FMCW beat signal as shown in (7) and $\boldsymbol{V}_{\boldsymbol{l}}$ is the diagonal matrix composed of the Doppler term, as follows:

$$
\boldsymbol{V}_{l}=\operatorname{diag}\left[v_{l}(0), v_{l}(1), \ldots, v_{l}(M-1)\right]
$$

where $\operatorname{diag}(\cdot)$ denotes a matrix operator and $\boldsymbol{v}_{\boldsymbol{l}}(\boldsymbol{m})$ is the $m$-th vector of the velocity term.

$$
\begin{gathered}
\tilde{y}_{l}(t)=\sum_{m=0}^{M-1}\left[\tilde{a}_{m} s_{\mathrm{tx}}\left(t-\tau_{m}\right) \exp \left(\frac{j 4 \pi l T_{F}}{\lambda}\left(x_{m, \mathrm{~h}}(t)+\sum_{p=1}^{P} x_{m, \mathrm{r}, p}(t)+\theta\right)\right)\right]+\omega(t) \\
y_{l}[n]=\sum_{m=0}^{M-1}[a_{m} \underbrace{\exp \left(j 2 \pi \mu \tau_{m} T_{s} n\right)}_{r\left(\tau_{m}\right), \text { range term }} \underbrace{\exp \left(j\left(\frac{4 \pi}{\lambda} l T_{F}\left(x_{m, h}+\sum_{p=1}^{P} x_{m, r, p}\right)+\theta\right)\right)}_{v_{l}(\mathrm{~m}) \text {, vital Doppler term }}]+\omega[n]
\end{gathered}
$$




\section{PROPOSED LOW-COMPLEXITY SUPER-RESOLUTION DE-}

\section{TECTION FOR RANGE-VITAL DOPPLER ESTIMATION RADAR}

This section proposes a low-complexity FFT-MUSIC algorithm based on target detection for range-vital Doppler estimation. This architecture is aimed at reducing the complexity to use the super resolution algorithm with accurate vital information. While conventional algorithms estimate the vital Doppler information of all targets, the proposed algorithm estimates only the target's vital Doppler information, as shown in Fig. 2.

\section{Distance Parameter Estimation by FFT}

First, the proposed algorithm finds the distance parameter of multiple targets from the FFT results of the received beat signal [13]. After the received signal is performed by the $1 \mathrm{D}-\mathrm{FFT}$, threshold detection is used to extract the distance index of various targets, and the FFT results corresponding to the distance index are acquired. Using threshold detection, the maximum peak value among the extracted FFT magnitude results is selected. As shown in Fig. 1, for the distance estimation, 1D-FFT [13] is performed on the received chirp signals with the obtained $l$-th chirp index. The 1D-FFT results $Y_{l}=\left[Y_{l}[0], Y_{l}\right.$ $\left.[1], \ldots, P_{l}[N-1]\right]^{\mathrm{T}}$ at the $l$-th chirp symbol, based on the distance information, are presented as:

$$
\boldsymbol{Y}_{l}=\boldsymbol{W}_{N} \boldsymbol{y}_{l}
$$

where $\boldsymbol{W}_{N}$ is a DFT matrix composed of $N$ column vectors with $N$ by 1 size, i.e., $\boldsymbol{W}_{N}=\left[W_{0}, W_{1}, \ldots, W_{N-1}\right]$, and the $u$-th column vector is denoted by $W_{u}$ and is expressed as

$$
W_{u}=\left[1, \exp \left(-j \frac{2 \pi u}{N}\right), \ldots, \exp \left(-j \frac{2 \pi u(N-1)}{N}\right)\right]^{\mathrm{T}}
$$

\section{Proposed Low Complexity Method for Vital Information}

In order to reject clutter information of the received signal, this proposed research uses the 1D-FFT results of multiple chirps of $Y_{1}, Y_{2, \ldots,} Y_{L}$. The phase variation detection using multiple chirp signals of each range sample can be obtained as follows:

$$
\boldsymbol{Y}_{l}^{\mathrm{PV}}=\boldsymbol{Y}_{l+1}-\boldsymbol{Y}_{l}
$$

Through phase variation detection of $1 \mathrm{D}-\mathrm{FFT}$, the range spectrum $I=\left[I_{1}, I_{2}, \ldots, I_{M}\right]$ peaks are extracted and the peak index is used to represent the range results of the targets. The phase variation detection results after range $1 \mathrm{D}-\mathrm{FFT}$ are applied to the super-resolution vital Doppler results.

When only the distance index is obtained by MUSIC [14], high-resolution vital Doppler information is acquired. When the positions of multiple targets are located differently, each target has a different Doppler component. The correlation matrix of the RX signal is set to full rank. However, when the vital components of each target are a coherent signal, the full rank of the matrix is nonexistent. This coherent signal's correlation matrix is a disadvantage. To solve this problem, a smoothing method is utilized to accurately detect vital Doppler. Using the FFT results of the detected $i$-th target $\boldsymbol{Y}_{\mathrm{Dopp}, i, q}=\left[Y_{q+1}^{P V}\left(I_{i}\right)\right.$, $\left.Y_{q+2}^{P V}\left(I_{i}\right), \ldots, Y_{q+Q}^{P V}\left(I_{i}\right)\right]^{T}$ that satisfy the conditions $Q \leq L$, the autocorrelation matrix $R_{X i}$ is formed as

$$
\boldsymbol{R}_{X}^{(i)}=\sum_{q=0}^{L-Q-1} \boldsymbol{P}_{\text {Dopp }, q}^{(i)} \boldsymbol{P}_{\text {Dopp }, q}^{(i) \mathrm{H}}
$$

The forward-backward technique is employed to reject the correlation effect, as in:

$$
\boldsymbol{R}_{f b}^{(i)}=\frac{1}{2}\left(\boldsymbol{R}_{X}^{(i)}+\boldsymbol{J} \boldsymbol{R}_{X}^{(i)} \boldsymbol{J}\right)
$$

where $J$ is the exchange matrix.

The forward-backward autocorrelation matrix $\boldsymbol{R}_{f b}^{(i)}$ is performed by the EVD [15] and is given as:

$$
\begin{aligned}
\boldsymbol{R}_{f b, i} & =\boldsymbol{T}\left[\boldsymbol{\Lambda}+\sigma^{2} \boldsymbol{I}\right] \boldsymbol{T}^{H} \\
& =\boldsymbol{T}\left[\begin{array}{ccccccc}
\lambda_{0}+\sigma^{2} & 0 & \cdots & 0 & 0 & \cdots & 0 \\
0 & \lambda_{1}+\sigma^{2} & \cdots & 0 & 0 & \cdots & 0 \\
\vdots & \vdots & \ddots & \vdots & \vdots & \vdots & \vdots \\
0 & 0 & \cdots & \lambda_{P-1}+\sigma^{2} & 0 & \cdots & 0 \\
0 & 0 & \cdots & 0 & \sigma^{2} & \cdots & 0 \\
\vdots & \vdots & \vdots & \vdots & \vdots & \ddots & \vdots \\
0 & 0 & \cdots & 0 & 0 & \cdots & \sigma^{2}
\end{array}\right] \boldsymbol{T}^{H}
\end{aligned}
$$

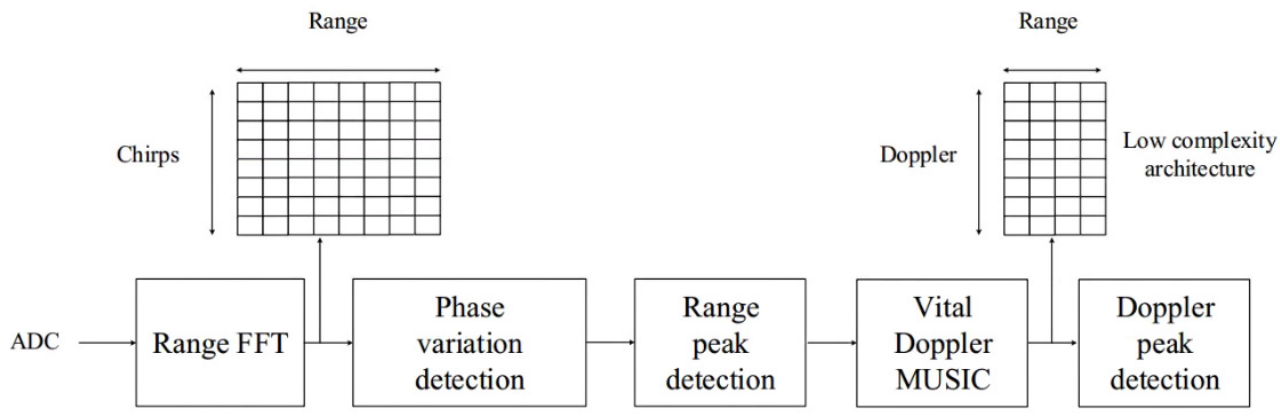

Fig. 2. Proposed architecture. 
where $T$ can divide the signal eigenvectors $Q$ with $P$ columns corresponding to the $P$ signal eigenvalues and noise eigenvectors $N$ with $T-P$ columns corresponding to the $T-P$ noise eigenvalues. The eigenvalues have a non-increasing order, i.e.,

$$
\lambda_{0} \geq \lambda_{1} \geq \cdots \geq \lambda_{P-1} \geq \lambda_{P}=\cdots \lambda_{Q-1}=\sigma^{2}
$$

The MUSIC algorithm is as follows. The vital Doppler vector $a(f)$ is matched with the signal subspace, and the columns of $N$ have orthogonality, such as:

$$
a(f) \boldsymbol{N}^{\mathrm{H}}=0
$$

where $a(f)$ is defined as:

$$
a(f)=\left[1, \exp \left(j 2 \pi f\left(T+T_{s}\right)\right), \ldots, \exp \left(j 2 \pi f(Q-1)\left(T+T_{s}\right)\right)\right]
$$

Using the principle of (18), in the $i$-th target among $M$ humans, the 1D-MUSIC spectrum can be determined as:

$$
P_{\text {MUSIC }}^{(i)}=\frac{1}{a(f) N^{\mathrm{H}} N a^{\mathrm{H}}(f)}
$$

Thus, the proposed parameter estimator for the vital Doppler for vital FMCW radars has been outlined.

\section{Summary of the Proposed Algorithm}

The major steps of the proposed algorithm are as follows:

Step 1: Using FFT results of the received beat signal, the proposed algorithm estimates the distance information of multiple targets.

Step 2: Through the phase variation detection of $1 \mathrm{D}-\mathrm{FFT}$, the maximum peaks among $M$ targets, with the exception of stationary targets, are achieved.

Step 3: After obtaining the distance index of the human target with vital Doppler, the magnitude and phase information of the distance index are obtained.

Step 4: When only the distance FFT signal corresponding to the distance index is stored, the total data size is reduced.

Step 5: Using the magnitude and phase information of only the distance FFT signal corresponding to the distance index, MUSIC is performed for super-resolution vital Doppler information.

\section{PERformance Evaluation By Simulation}

In this section, performance of the proposed algorithm, estimated in various simulations, is compared with that of conventional algorithms, such as FFT and MUSIC.

\section{Simulation Environment}

To assess the performance of the proposed algorithm, two simulations were performed. First, the spectrum results of the proposed algorithm and of full search FFT-MUSIC for vital information are obtained. In the second simulation, the rootmean-square error (RMSE) of the algorithms is determined based on the difference of the distance for the two targets. The SNR is defined by SNR $=10 \log _{10}\left(\sigma_{s}^{2} / \sigma_{w}^{2}\right)$, where $\sigma_{s}^{2}$ denotes the power of the signal, and $\sigma_{w}^{2}$ is the noise power. The FMCW radar simulation parameters are listed in Table 1. The RMSE values according to the parameters of each algorithm at various SNR are calculated $N_{T}$ times for the received signals. The RMSE is defined by RMSE $=\sqrt{\frac{1}{N_{T}} \sum_{n=1}^{N_{T}}\left(\hat{\theta}_{n}-\theta\right)}, \mathrm{n} N_{T}$ is set to $10^{3}$, and $\hat{\theta}_{n}$ is the estimated angle of the target in the $n$ th Monte-Carlo trial.

\section{Simulation Results}

When we consider two humans who are different distances from a radar, they can be extracted in terms of vital information by both the proposed algorithm and the conventional algorithms, while the complexity of the proposed method is lower than that of conventional methods. In Fig. 3, two targets are located at $3.75 \mathrm{~m}$ and $6 \mathrm{~m}$. The simulated respiration and heartbeat signals were set to 18 beats $/ \mathrm{min}(0.3 \mathrm{~Hz})$ and 63 beats $/ \mathrm{min}$ $(1.05 \mathrm{~Hz})$, respectively, and the vital information was included with amplitudes satisfying the following: $a_{1, \mathrm{r}}: a_{1, \mathrm{~h}}=10: 2$. The simulation performance was compared using the spectral results of the full search FFT-MUSIC and proposed algorithms. The

Table 1. Summary of simulation environment

\begin{tabular}{lc}
\hline \multicolumn{1}{c}{ Parameter } & Value \\
\hline Center frequency & $24 \mathrm{GHz}$ \\
Bandwidth & $200 \mathrm{MHz}$ \\
Chirp duration $(T)$ & $80 \mu \mathrm{s}$ \\
Chirp-to-chirp interval $(T i)$ & $150 \mathrm{~ms}$ \\
Number of samples per chirp $(N s)$ & 80 \\
Number of chirps per frame $(L)$ & 256 \\
\hline
\end{tabular}

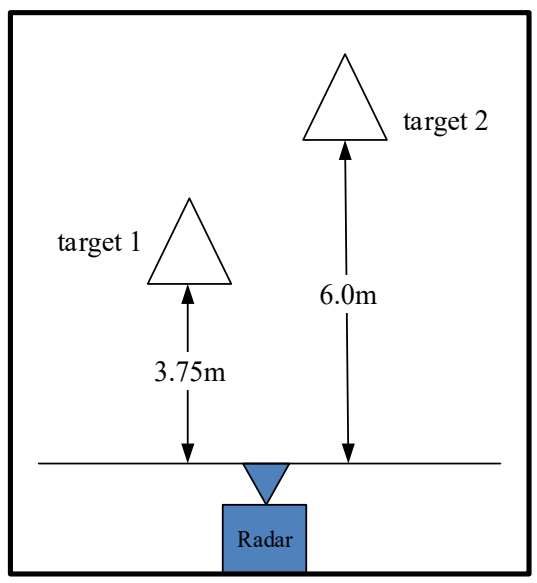

Fig. 3. Simulation environment of two targets. 
SNR was set to $20 \mathrm{~dB}$. Fig. 3 shows the simulation environment of the two targets.

Fig. 4 shows a comparison of each algorithm's distance-vital Doppler spectrum. The conventional full search FFT-MUSIC in Fig. 4(a) and the proposed algorithm in Fig. 4(b) have the same estimation results, while the conventional full search algorithm has higher complexity than those of the proposed algorithms. This is the reason to choose the target signal instead of performing a full search for vital information estimation. Our algorithm has a 193 beats/min maximum heartbeat as the chirpto-chirp interval ( $T i)$ is $150 \mathrm{~ms}$ and chirp duration $(T)$ is $80 \mu \mathrm{s}$. The interval of chirps is inversely proportional to maximum Doppler range in [13].

Fig. 5 provides a comparison between the proposed and conventional algorithms for RMSE according to various SNR. In Fig. 5, conventional algorithms include the FFT [13], MUSIC [15], and extrapolation [16]. The estimation performance of the first target in the presence of the second target is focused on. In terms of the distance parameter in Fig. 5(a), when the SNR varies from 0 to $20 \mathrm{~dB}$, the RMSE of the distance estimation of the first target is shown. In Fig. 5(a), with the wide distance difference of $2.25 \mathrm{~m}$, the RMSEs of both the proposed and the conventional schemes, such as FFT, are similar. When the vital information of two persons is similar, as in Fig. 5(b), the proposed structure has results similar to those of the MUSIC algorithm under all SNRs, while the proposed algorithm has lower complexity.

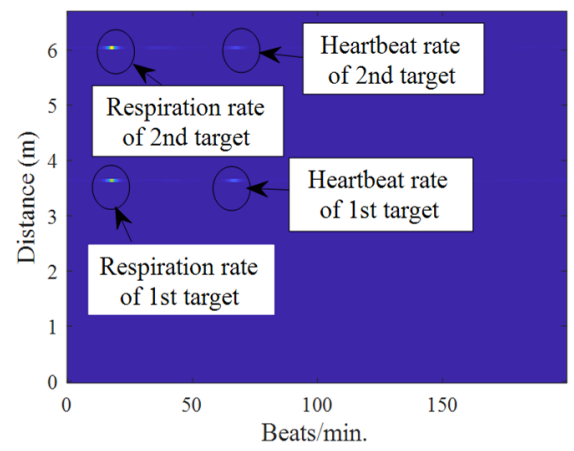

(a)

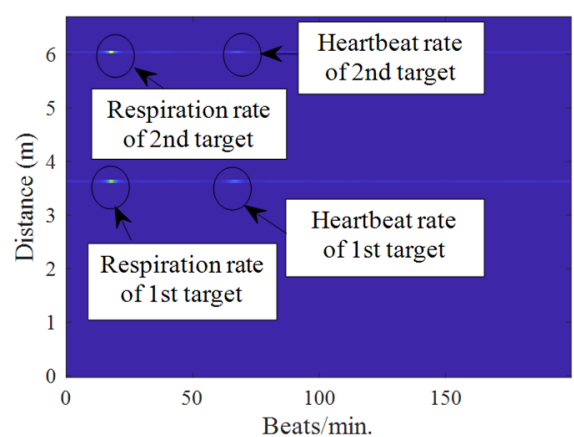

(b)

Fig. 4. Each algorithm's distance-vital Doppler spectrum: (a) full search FFT-MUSIC and (b) proposed algorithm.

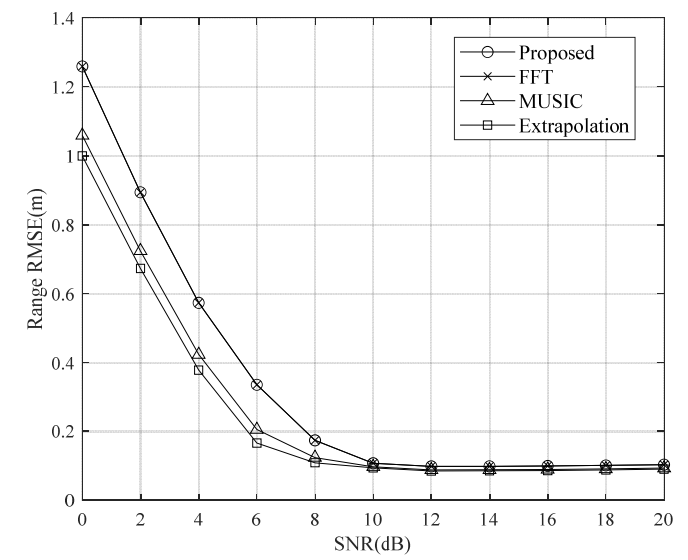

(a)

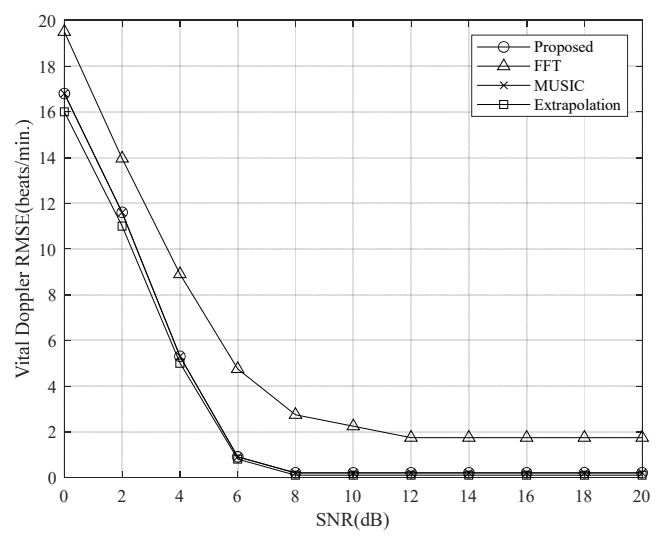

(b)

Fig. 5. RMSEs versus (a) distance difference and (b) vital Doppler parameter.

\section{Complexity Analysis}

In this section, the proposed algorithm and 2D-MUSIC are subjected to complexity analysis. The computational complexity of the algorithms consists of the primary multiplication operation. 2D-MUSIC uses full search to detect human targets for vital Doppler and distances, whereas the proposed algorithm requires a memory-efficient search to estimate multiple parameters. The 2D-MUSIC algorithm's complexity is composed of an autocorrelation matrix, EVD, and spectrum generation by the orthogonality, as shown in (21). In (22), $L$ times $N$-point FFT for distance estimation and $N$ times $L$-point FFT for vital Doppler estimation is represented. (23) shows that 2D-extrapolation's complexity is analyzed using prediction order $(p)$ and the number of samples being extrapolated $(O)$. In the case of the proposed algorithm, $L$ times $N$-point FFT for distance estimation and $M$ times $1 \mathrm{D}-\mathrm{MUSIC}$, which only has the target's data for vital Doppler estimation, are performed as shown in (24). Here, $R$ denotes the number of spectrum samples of the MUSIC algorithm. When the complexity of the proposed structure in (24) is compared with that of 2D-MUSIC, 2D-FFT, and 2Dextrapolation in (21)-(23), the proposed structure in (24) has a complexity of $N^{3}$ while 2D-FFT serves as a complexity of $N L$, 


$$
\begin{gathered}
C_{2 \text { D-MUSIC }}=M N^{2} L^{2}+N^{3} L^{3}+R^{2}[N L(N L-M)+N L-M] \\
C_{2 \text { D-FFT }}=L N \log _{2}(N)+N L \log _{2}(L) \\
C_{2 \text { D-extra. }}=6 L N p-p^{2}+p O(L+N) \\
C_{\text {proposed }}=L N \log _{2}(N)+M\left(\left(\frac{N^{2}}{2}+\frac{N}{2}\right)+\frac{16 N^{3}}{2}+\left(\frac{(N-M) N^{2}}{2}+\frac{(N-M) N}{2}\right)+R\left(N^{2}+N\right)\right)
\end{gathered}
$$

2D-extrapolation as a complexity of $N L p$, and 2D-MUSIC as a complexity of $N^{3} L^{3}$. The complexities of the 2D-FFT, 2Dextrapolation, 2D-MUSIC, and the proposed algorithms are denoted by $C_{2 \mathrm{D}-\mathrm{FFT}}, C_{2 \mathrm{D}-\text { extra. }}, C_{2 \mathrm{D}-\mathrm{MUSIC}}$, and $C_{\text {proposed }}$. They are calculated in (21)-(24).

Fig. 6 provides a comparison of the primary multiplication operations for the proposed algorithm and 2D-MUSIC. When the numbers of received samples $N$ are 16 and 64, respectively, Fig. 6 shows the required number of multiplications according to the various numbers of chirp symbols. In Fig. 6(a), when the number of chirp signals $L$ is 16 , the complexity of the proposed algorithm is about $4.6 \times 10^{6}$ greater than that of $2 \mathrm{D}-\mathrm{MUSIC}$. In Fig. 6(b), it can be seen that when the number of chirp signals $L$ is 64 , the complexity of the proposed algorithm is about $2.4 \times 10^{7}$ greater than that of $2 \mathrm{D}$-MUSIC. Therefore, the

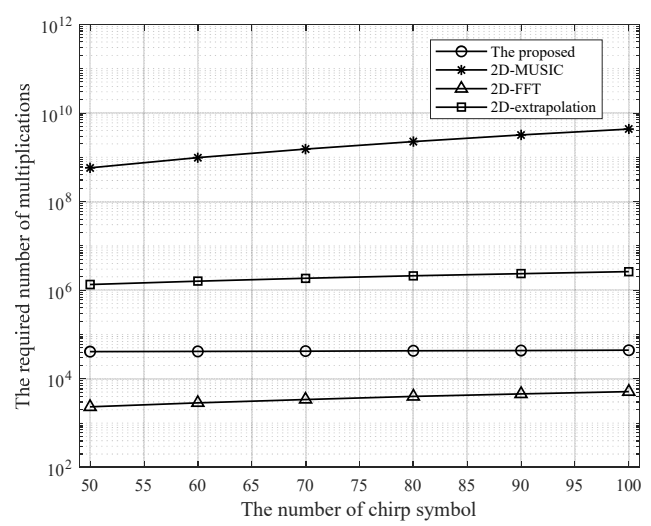

(a)

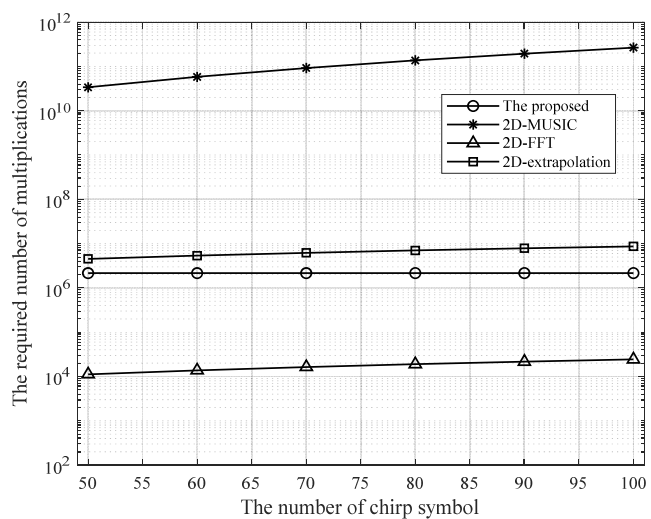

(b)

Fig. 6. Complexity analysis results: (a) number of chirp symbols $L=16$ and (b) number of chirp symbols $L=64$. complexity of the proposed algorithm is greatly reduced compared to that of 2D-MUSIC.

\section{EXPERIMENTS}

Experiment results were established in an indoor experimental laboratory in South Korea. In Fig. 7, the overall experimental environment is represented. The RF parameters are provided in Table 1 . With a $24 \mathrm{GHz}$ center frequency, a FMCW RF module with 1 TX channel and 3 RX channels was involved. The TX consisted of a frequency synthesizer, a voltage-controlled oscillator, and an oscillator with $26 \mathrm{MHz}$. An FMCW signal was generated for the $200 \mathrm{MHz}$ bandwidth in a range of 24.0524.25 GHz with $8 \mathrm{dBm}$ output power as shown in Fig. 8 [10]. This multi-patch antenna has a gain of $15.6 \mathrm{dBi}$. An RF signal is moved to the TX antenna and receiver mixer via the power divider. The power divider with an $S_{21}$ parameter of $-6 \mathrm{~dB}$, an $S_{31}$ parameter of $-1.5 \mathrm{~dB}$, and an $S_{11}$ parameter of $-20 \mathrm{~dB}$ were used.

The receiver consisted of two high-pass filters (HPFs), two low-pass filters (LPFs), two low-noise amplifiers (LNAs), and two mixers. The receiver had an overall noise figure of $8 \mathrm{~dB}$. The gain and the noise figure of the LNAs were $14 \mathrm{~dB}$ and $2.5 \mathrm{~dB}$, respectively. An RF signal was down-converted to an intermediate frequency (IF) signal (beat signal) by the mixer. The measured $3 \mathrm{~dB}$ cutoff frequency of the HPFs and the LPFs are approximately $13 \mathrm{kHz}$ and $2 \mathrm{MHz}$, respectively. The developed 24 GHz FMCW RF module is shown in Fig. 9 [10]. The reference equipment was a watch-type monitoring device, i.e., a Xiaomi Mi Band 2.

When two targets were together in an indoor room, the ex-

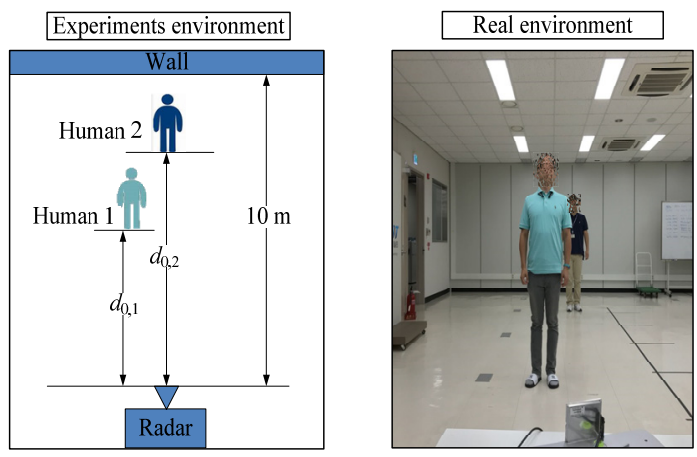

Fig. 7. Indoor experiment environment with two humans. 


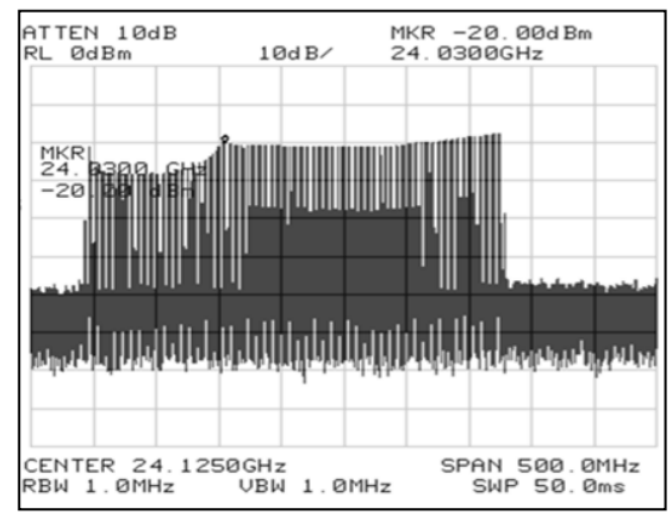

Fig. 8. Measured TX signal with a range of 24.025-24.225 GHz. Adapted from [10] with permission of the IEEE.

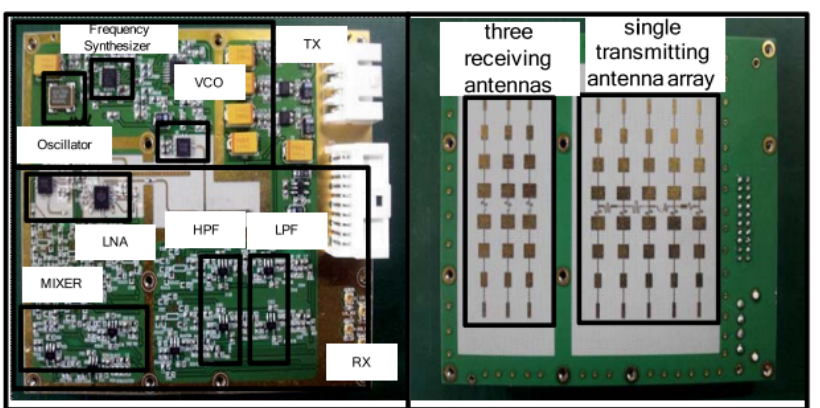

Fig. 9. Photograph of the $24 \mathrm{GHz}$ FMCW radar sensor system. Adapted from [10] with permission of the IEEE.

perimental estimation results obtained using these algorithms were compared with the corresponding reference signal. In this part, the same parameters are required as in the simulation. In addition, experiments for two cases are performed, as shown in Table 2. Two far-field targets, such as humans, were located at $d_{0,1}=2.9 \mathrm{~m}$ and $d_{0,2}=4.9 \mathrm{~m}$ in Case I and $d_{0,1}=2.9 \mathrm{~m}$ and $d_{0,2}=$ $5.4 \mathrm{~m}$ in Case II.

In terms of Case I, when the two humans were located at a distance difference of $2.5 \mathrm{~m}$, the FFT results with MTI and the proposed results with MTI were able to separate the two humans simultaneously, as shown in Fig. 10. Due to the distance resolution of $2.0 \mathrm{~m}$ at this bandwidth, the experimental results with distance difference over $2.5 \mathrm{~m}$ show that the conventional algorithms and proposed algorithm can resolve the two targets.

In Case II, the FFT results and the proposed FFT-MUSIC results simultaneously detect two persons, as shown in Fig. 11. Through an experimental comparison of the FFT and proposed algorithms, the conventional and proposed algorithms have similar performance.

Table 2. Distance difference conditions (unit: $\mathrm{m}$ )

\begin{tabular}{lccc}
\hline & $d_{0,1}$ & $d_{0,2}$ & $\left|d_{0,2}-d_{0,1}\right|$ \\
\hline Case I & 2.9 & 5.4 & 2.5 \\
Case II & 2.9 & 4.9 & 2.0 \\
\hline
\end{tabular}

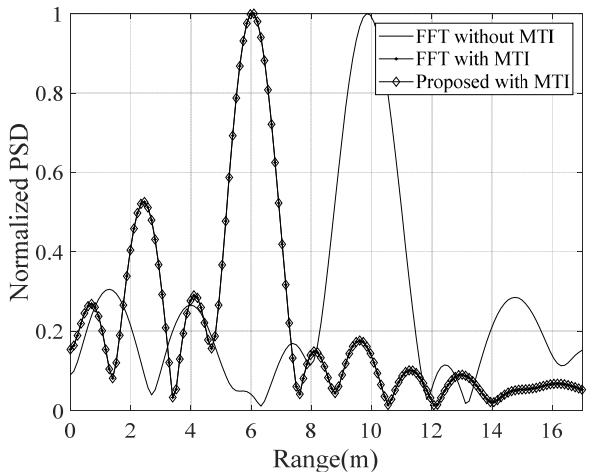

(a)

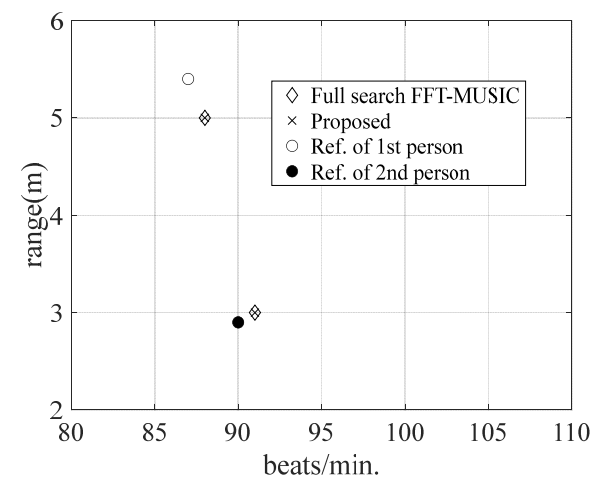

(b)

Fig. 10. Experimental results: (a) of $1 \mathrm{D}$ range spectrum and (b) range-Doppler map for Case I.

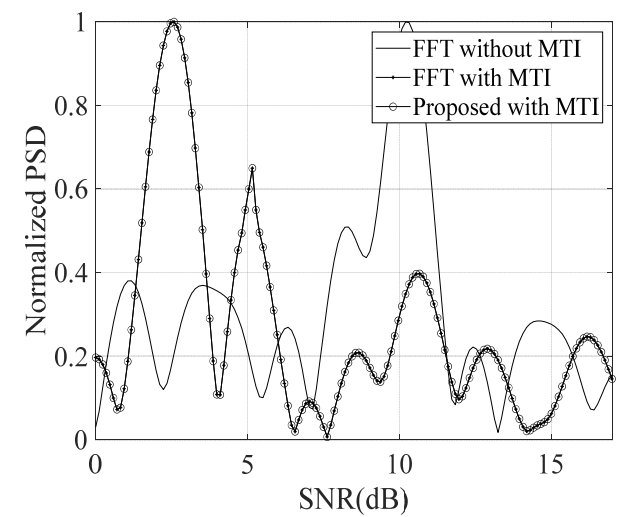

(a)

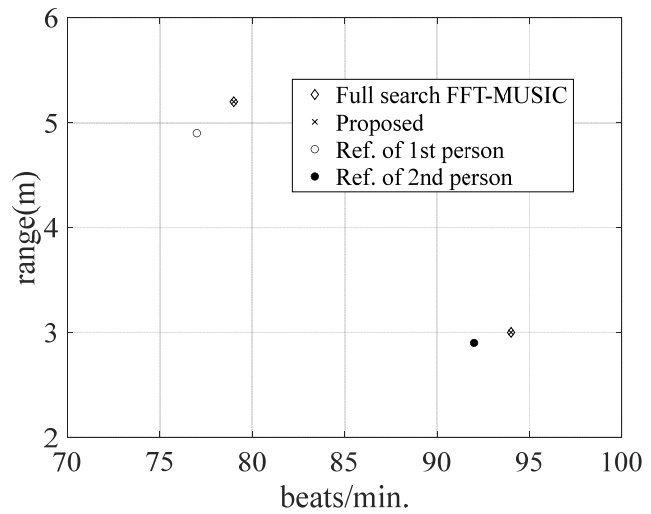

(b)

Fig. 11. Experimental results: (a) of $1 \mathrm{D}$ range spectrum and (b) range-Doppler map for Case II. 


\section{CONCLUSION}

This paper proposed the low-complexity FFT-MUSIC algorithm based on target detection for a range-vital Doppler estimator of contactless vital FMCW radar. To improve the accuracy of the parameters for the vital radar, a high resolution-based algorithm was proposed in the form of MUSIC. However, the high resolution-based vital radar is difficult to apply to low-cost and real-time vital FMCW systems. As only target information and vital information through distance FFT by phase variation detection is extracted, the low complexity FFT-MUSIC can be obtained. In cases of complexity, when the number of chirp signals $L$ are 16 and 64, the complexity values of the proposed algorithm are approximately $4.6 \times 10^{6}$ and $2.4 \times 10^{7}$ greater than that of the 2D-MUSIC, respectively. Through simulation and experimental comparisons of the full search FFT-MUSIC and proposed algorithms, the conventional and proposed algorithms are found to have similar performance. In the future, these results will be utilized in an embedded system to enhance the parameter accuracy.

This work was supported by the DGIST R\&D Program of the Ministry of Science, ICT and Future Planning, Korea (No. 21-IT-02) and the Technology Development Program funded by the Ministry of SMEs and Startups, Korea (No. S2987884).

\section{REFERENCES}

[1] C. Li and J. Lin, "Random body movement cancellation in Doppler radar vital sign detection," IEEE Transactions on Microwave Theory and Techniques, vol. 56, no. 12, pp. 31433152, 2008.

[2] Z. Peng and C. Li, "Portable microwave radar systems for short-range localization and life tracking: a review," Sensors, vol. 19, no. 5, article no. 1136, 2019. https://doi.org/ $10.3390 / \mathrm{s} 19051136$

[3] L. Sun, Y. Li, H. Hong, F. Xi, W. Cai, and X. Zhu, "Superresolution spectral estimation in short-time non-contact vital sign measurement," Review of Scientific Instruments, vol. 86, no. 4 , article no. 044708 , 2015. https://doi.org/10.1063 $/ 1.4916954$

[4] J. C. Lin, "Noninvasive microwave measurement of respiration," Proceedings of the IEEE, vol. 63, no. 10, pp. 1530-1530, 1975.

[5] K. M. Chen, D. Misra, H. Wang, H. R. Chuang, and E. Postow, "An X-band microwave life-detection system,"
IEEE Transactions on Biomedical Engineering, vol. 33, no. 7, pp. 697-701, 1986.

[6] C. Li, Y. Xiao, and J. Lin, "Experiment and spectral analysis of a low-power Ka-band heartbeat detector measuring from four sides of a human body," IEEE Transactions on Microwave Theory and Techniques, vol. 54, no. 12, pp. 4464-4471, 2006.

[7] J. C. Lin, "Microwave sensing of physiological movement and volume change: a review," Bioelectromagnetics, vol. 13, no. 6, pp. 557-565, 1992.

[8] K. M. Chen, Y. Huang, J. Zhang, and A. Norman, "Microwave life-detection systems for searching human subjects under earthquake rubble or behind barrier," IEEE Transactions on Biomedical Engineering, vol. 47, no. 1, pp. 105-114, 2000.

[9] Z. Peng, J. M. Munoz-Ferreras, Y. Tang, C. Liu, R. GomezGarcia, L. Ran, and C. Li, "A portable FMCW interferometry radar with programmable low-IF architecture for localization, ISAR imaging, and vital sign tracking," IEEE Transactions on Microwave Theory and Techniques, vol. 65, no. 4, pp. 1334-1344, 2017.

[10] D. Oh and J. H. Lee, "Low-complexity range-azimuth FMCW radar sensor using joint angle and delay estimation without SVD and EVD," IEEE Sensors Journal, vol. 15, no. 9, pp. 4799-4811, 2015.

[11] X. Zhang and D. Xu, "Low-complexity ESPRIT-based DOA estimation for colocated MIMO radar using reduced-dimension transformation," Electronics Letters, vol. 47, no. 4, pp. 283-284, 2011.

[12] X. Nie and L. Li, "A computationally efficient subspace algorithm for 2-D DOA estimation with L-shaped array," IEEE Signal Processing Letters, vol. 21, no. 8, pp. 971-974, 2014.

[13] S. Kim, D. Oh, and J. Lee, "Joint DFT-ESPRIT estimation for TOA and DOA in vehicle FMCW radars," IEEE Antennas and Wireless Propagation Letters, vol.14, pp. 17101713, 2015.

[14] P. Stoica and A. Nehorai, "MUSIC, maximum likelihood, and Cramer-Rao bound: further results and comparisons," IEEE Transactions on Acoustics, Speech, and Signal Processing, vol.38, no. 12, pp. 2140-2150, 1990.

[15] C. L. Liu and P. P. Vaidyanathan, "Remarks on the spatial smoothing step in coarray MUSIC," IEEE Signal Processing Letters, vol. 22, no. 9, pp.1438-1442, 2015.

[16] S. Kim, B. S. Kim, Y. Jin, and J. Lee, "ExtrapolationRELAX estimator based on spectrum partitioning for DOA estimation of FMCW radar," IEEE Access, vol. 7, pp. 98771-98780, 2019. 


\section{Bongseok Kim}

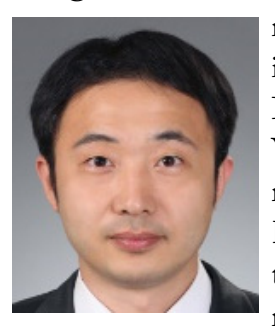

received his B.S. degree in Electronics Engineering in 2006 as well as his M.S. and Ph.D. degrees in Information and Communications Engineering from Yeungnam University, Korea in 2009 and 2014, respectively. Since 2014, he has worked at the DGIST, Korea as a senior research engineer. His current interests include multi-functional radar systems and radar signal processing.

\section{Youngseok Jin}

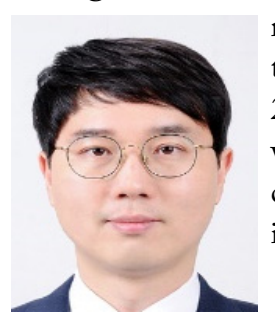

received his B.S. and M.S. degrees in Communication Engineering from Daegu University, Korea in 2010 and 2012, respectively. Since 2012, he has worked as a researcher at the DGIST, Korea. His current interests include radar signal processing and implementation in FPGA/DSP.

\section{Youngdoo Choi}

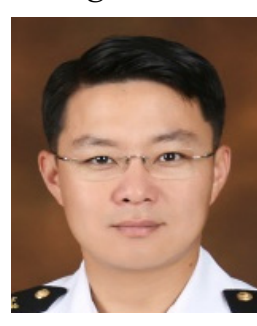

received his B.S. degree in Underwater Acoustics from the ROK naval Academy, Jinhae, South Gyeongsang Province, South Korea in 2003. He went on to receive his M.S. degree in Electronics Engineering from Kyungpook National University, Daegu, South Korea in 2013 and a Ph.D. degree in Electronics Engineering from Kyungpook National University, Daegu, South Korea in 2016. From 2003, he has worked as the ROK Navy Officer, South Korea. From 2017 to 2020, he was in charge of education control at the ROK Joint Military College of Daejeon, South Korea. From 2020, he has also worked as an Assistant Professor at the ROK navy academy. His research interests include SONAR, detection algorithms, and underwater signal processing.

Jonghun Lee

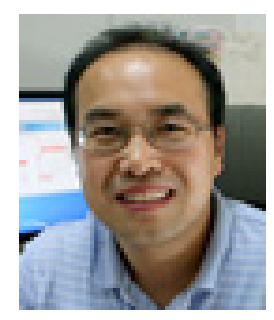

received his B.S. degree in Electronics Engineering from Sungkyunkwan University, Korea in 1996, as well as his M.S. and Ph.D. degrees in Electrical and Electronics and Computer Science from Sungkyunkwan University, Korea in 1998 and 2002, respectively. From 2002 to 2005, he worked at the Samsung Electronics Company as a principal research engineer. Since 2005, he has worked as a director $\&$ principal researcher in the Division of Automotive Technology at the DGIST, South Korea. He has also worked as a full professor with the Department of Interdisciplinary Engineering at the DGIST. He is an IEEE senior member. His primary research interests are detection, tracking, recognition for radar (FMCW \& UWB radar), radar based vehicle sensors, radar signal processing, and ML/DL for sensor fusion.

\section{Sangdong Kim}

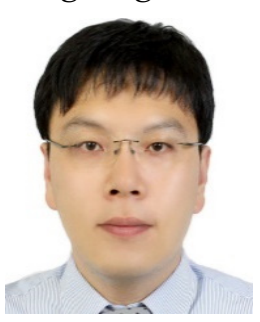

received a B.S. degree in School of Electrical and Computer from Hanyang University in 2004, an M.S. degree in Department of Electronic, Communication and Computer Engineering from Hanyang University in 2006, and a Ph.D. degree in Electronics Engineering from Kyungpook National University in 2018. From 2015 to 2016, he was a Visiting Scholar at the University of Florida. Since 2006, he worked as a Principal Researcher with the Division of Automotive Technology at the DGIST in Daegu, South Korea. Since 2020, he has also worked as an Assistant Professor with the Interdisciplinary Engineering at the DGIST. His research interests include super-resolution algorithms, automotive radar, and vital radar. 